\title{
The magnetic fields of large Virgo Cluster spirals ${ }^{\star}$
}

\author{
M. Weżgowiec ${ }^{1}$, M. Urbanik ${ }^{1}$, B. Vollmer ${ }^{2}$, R. Beck ${ }^{3}$, K. T. Chyży ${ }^{1}$, M. Soida ${ }^{1}$, and Ch. Balkowski ${ }^{4}$ \\ 1 Obserwatorium Astronomiczne Uniwersytetu Jagiellońskiego, ul. Orla 171, 30-244 Kraków, Poland \\ e-mail: markmet@oa.uj .edu.pl \\ 2 CDS, Observatoire Astronomique de Strasbourg, UMR 7550, 11 rue de l'université, 67000 Strasbourg, France \\ ${ }_{3}$ Max-Planck-Institut für Radioastronomie, Auf dem Hügel 69, 53121 Bonn, Germany \\ ${ }^{4}$ Observatoire de Paris, GEPI, CNRS, and Université Paris 7, 5 Place Jules Janssen, 92195 Meudon Cedex, France
}

Received 19 December 2006 / Accepted 28 April 2007

\begin{abstract}
Context. Because of its proximity the Virgo Cluster is an excellent target for studying interactions of galaxies with the cluster environment. Both the high-velocity tidal interactions and effects of ram pressure stripping by the intracluster gas can be investigated. Aims. Optical and/or H I observations do not always show the effects of weak interactions between galaxies and their encounters with the cluster medium. For this reason we searched for possible anomalies in the magnetic field structure in Virgo Cluster spirals that could be attributed to perturbations in their gas distribution and kinematics.

Methods. Five angularly large Virgo Cluster spiral galaxies (NGC 4501, NGC 4438, NGC 4535, NGC 4548, and NGC 4654) were the targets of a sensitive total power and polarization study using the $100-\mathrm{m}$ radio telescope in Effelsberg at 4.85 GHz. For two objects, polarization data at higher frequencies were obtained allowing Faraday rotation analysis.

Results. Distorted magnetic field structures were identified in all galaxies. Interaction-induced magnetized outflows were found in NGC 4438 (due to nuclear activity) and NGC 4654 (a combination of tidal tails and ram pressure effects). Almost all objects (except the anaemic NGC 4548), exhibit distortions in polarized radio continuum attributable to the influence of the ambient gas. For some galaxies they agree with observations of other species, but the magnetic field is sometimes (NGC 4535) the only tracer of the interaction with the cluster environment.

Conclusions. The cluster environment clearly affects the evolution of the galaxies due to ram pressure and tidal effects. Magnetic fields provide a very long lasting memory of past interactions. Therefore, they are a good tracer of weak interactions that are difficult to detect by other observations. Information about motions of galaxies in the sky plane and their three-dimensional distribution can also be obtained.
\end{abstract}

Key words. galaxies: clusters: individual: Virgo - galaxies: magnetic fields - radio continuum: galaxies

\section{Introduction}

Cluster galaxies are known to interact intensively with the intracluster medium (ICM). One of the effects is the ram pressure stripping first discussed by Gunn \& Gott (1972). This process is particularly active in central parts of a cluster with highdensity ICM, giving rise to ram pressure effects and stripping of the outer parts of the gaseous disks of galaxies (Vollmer et al. 2001, 2004a). This leads to significant H I deficiencies in such objects (Cayatte et al. 1990, 1994). These interactions seem to influence only the neutral and the ionized gas (Chemin et al. 2006), while the observations of stellar populations (Gavazzi et al. 1991, 1998) and of CO content (Boselli et al. 1997) show no traces of ram pressure effects. However, tidal interactions (see Toomre \& Toomre 1972) may also be important for cluster galaxies, as their relative distances are about 10 times shorter than between field objects. The cluster galaxies can interact either with the cluster potential (Byrd \& Valtonen 1990; Valluri 1993) or with other cluster galaxies ("galaxy harassment", Moore et al. 1998). Special attention should be given to the high-velocity tidal encounters that occur almost exclusively in the cluster environment. Due to the high

^ Based on the observations with the 100-m telescope at Effelsberg operated by the Max-Planck-Institut für Radioastronomie (MPIfR) on behalf of the Max-Planck-Gesellschaft. relative velocities between the cluster galaxies, a small impact parameter is required to perturb them efficiently. The probability of such encounters is rather low. We also note that, in contrast to ram pressure effects, tidal interactions affect both the gas and stellar content of a galaxy.

The interactions between the interstellar medium of the galaxies (ISM) and the ICM are already known to cause strong gas compression effects, observed as $\mathrm{HI}$ ridges in the outskirts of galactic disks (Cayatte et al. 1990). Observations of the polarized emission in perturbed galaxies are known to provide a sensitive tool for tracing the anomalous gas motions and compression effects, even when the disturbances are hardly visible in other species (Soida et al. 2001). Moreover, for cluster galaxies a unique possibility of determining the directions of the gas flows in the sky plane arises, adding a new dimension to the radial velocity studies (Urbanik 2005).

Due to its proximity and a high content of spiral galaxies, the Virgo Cluster is the best target for observing the effects of both kinds of interactions. It is the nearest cluster of galaxies, situated at a distance of only $17 \mathrm{Mpc}$, which enables a sufficient resolution even with single-dish radio telescopes. The cluster covers an area of approx. $10 \times 10^{\circ}(3 \times 3 \mathrm{Mpc}$ in the sky plane) with an even greater extent towards the southern extension. The main subcluster concentrates around M 87 and is characterised by a high density of the ICM as seen in X-ray maps of 
Table 1. Basic astronomical properties and parameters of radio observations of the studied galaxies.

\begin{tabular}{|c|c|c|c|c|c|c|c|c|c|}
\hline \multirow[t]{3}{*}{$\overline{\mathrm{NGC}}$} & \multirow{3}{*}{$\begin{array}{l}\text { Morph. } \\
\text { type }^{a}\end{array}$} & \multicolumn{2}{|c|}{ Optical position $^{a}$} & \multirow{3}{*}{$\begin{array}{c}\text { Incl. }^{a} \\
{\left[{ }^{\circ}\right]}\end{array}$} & \multirow{3}{*}{$\begin{array}{c}\text { Pos. } \\
\text { ang. }{ }^{a}\left[{ }^{\circ}\right]\end{array}$} & \multirow{3}{*}{$\begin{array}{l}\text { Dist. to } \\
\left.\text { Vir A [ }{ }^{\circ}\right]\end{array}$} & \multirow{3}{*}{$\begin{array}{l}\text { Number } \\
\text { of cov. }\end{array}$} & \multirow{2}{*}{\multicolumn{2}{|c|}{$\begin{array}{c}\text { rms in final map } \\
{[\mathrm{mJy} / \mathrm{b} . \mathrm{a} .]}\end{array}$}} \\
\hline & & \multirow[t]{2}{*}{$\alpha_{2000}$} & \multirow[t]{2}{*}{$\delta_{2000}$} & & & & & & \\
\hline & & & & & & & & TP & PI \\
\hline 4438 & $\mathrm{Sa}$ & $12^{\mathrm{h}} 27^{\mathrm{m}} 45^{\mathrm{s}} .9$ & $+13^{\circ} 00^{\prime} 32^{\prime \prime} .2$ & 90 & 27 & 0.9 & 25 & 0.7 & 0.07 \\
\hline \multirow[t]{2}{*}{4501} & $\mathrm{Sb}$ & $12^{\mathrm{h}} 31^{\mathrm{m}} 59^{\mathrm{s}} 3$ & $+14^{\circ} 25^{\prime} 13^{\prime \prime} \cdot 7$ & 60 & 138 & 2 & 12 & 0.9 & 0.09 \\
\hline & & & & & & & $23^{b}$ & $0.4^{b}$ & $0.17^{b}$ \\
\hline 4535 & $\mathrm{SBc}$ & $12^{\mathrm{h}} 34^{\mathrm{m}} 20^{\mathrm{s}} \cdot 4$ & $+08^{\circ} 11^{\prime} 52^{\prime \prime} .3$ & 41.3 & 180 & 4.3 & 10 & 0.7 & 0.1 \\
\hline 4548 & $\mathrm{SBb}$ & $12^{\mathrm{h}} 35^{\mathrm{m}} 26^{\mathrm{s}} .4$ & $+14^{\circ} 29^{\prime} 47^{\prime \prime} 0$ & 35 & 150 & 2.4 & 11 & 0.8 & 0.09 \\
\hline \multirow[t]{3}{*}{4654} & $\mathrm{SBc}$ & $12^{\mathrm{h}} 43^{\mathrm{m}} 57^{\mathrm{s}} .0$ & $+13^{\circ} 07^{\prime} 34^{\prime \prime} 3$ & 57.7 & 122 & 3.3 & 15 & 0.6 & 0.06 \\
\hline & & & & & & & $33^{c}$ & $0.25^{c}$ & $0.03^{c}$ \\
\hline & & & & & & & $31^{b}$ & $0.6^{b}$ & $0.14^{b}$ \\
\hline
\end{tabular}

${ }^{a}$ Taken from HYPERLEDA database - http://leda.univ-lyon1.fr - see Paturel et al. (1983). ${ }^{b}$ At 10.45 GHz. ${ }^{c}$ At 8.35 GHz.

Böhringer (1994). The southern extension of the cluster forms another subcluster concentrated around the giant elliptical galaxy M49. It is smaller and less dense and is associated with a distinct concentration of hot gas seen in the X-ray maps (Böhringer 1994). More distinct subclusters have been identified by Gavazzi (1999).

So far, detailed information on possible effects of the cluster environment on the galactic magnetic fields is restricted to three Virgo Cluster objects. In the first one - NGC 4254, only weakly affected by the ambient gas - a strongly polarized region on the disk side facing the cluster core has been found (Chyży et al. 2002; Soida et al. 1996). In the highly inclined NGC 4522, the polarized emission is asymmetric, with extension coinciding with extraplanar $\mathrm{H} \alpha$ emission (Vollmer at al. 2004b). The third galaxy - NGC 4569 - was found to possess large vertical polarized radio lobes, at least one of which also shows signs of external compression (Chyży et al. 2006).

In order to obtain comprehensive information on global magnetic field structures of cluster spirals undergoing various types of interactions with the cluster environment, we performed the first systematic study of the radio polarization of five angularly large Virgo Cluster spirals with the Effelsberg 100-m radio telescope. To search for large-scale outflows or radio tails, we observed all objects in total power and polarization at $4.85 \mathrm{GHz}$ with a beam of 2.5 ( $1^{\prime}$ corresponds to about $5 \mathrm{kpc}$ at the distance to the Virgo Cluster). Objects with bright enough radio disks were also observed at $10.45 \mathrm{GHz}$ or at $8.35 \mathrm{GHz}$ (beams of 1.13 , and 1.5 , resp.), in order to obtain more details of the magnetic field structure and Faraday rotation properties. We chose the following galaxies:

- NGC 4438 - a strongly stripped galaxy in the Virgo Cluster centre (Chemin et al. 2005), also perturbed by tidal forces from its companion (Vollmer et al. 2005).

- NGC 4501 - a galaxy showing significant (but not extreme) effects of gas stripping (Cayatte et al. 1990, 1994; Chemin et al. 2006) and a strong asymmetry in the H I distribution. It is situated close to an X-ray cloud found by Böhringer et al. (1994).

- NGC 4535 - a relatively unperturbed, optically symmetric grand-design spiral in the southern extension of the Virgo Cluster with a fairly symmetric H I distribution (Cayatte et al. 1990; Chemin et al. 2006).

- NGC 4548 - an anaemic galaxy with a strong H I deficiency and a low star formation level (Cayatte et al. 1990, 1994; Chemin et al. 2006).

- NGC4654 - a mildly stripped galaxy (Cayatte et al. 1990, 1994; Chemin et al. 2006) with cometary appearance and an $\mathrm{H}$ I tail probably caused by joint effects of tidal interactions with NGC4639 and ram pressure stripping (Vollmer 2003; Phookun \& Mundy 1995).

\section{Observations and data reduction}

The observations were performed between March 2001 and May 2003 using the 100-m Effelsberg radio telescope of the MaxPlanck-Institut für Radioastronomie ${ }^{1}$ (MPIfR) in Bonn. The basic astronomical properties of the observed objects and of our observations are summarised in Table 1.

All galaxies were observed at $4.85 \mathrm{GHz}$ using the twohorn system (with horn separation of $8^{\prime}$ ) in the secondary focus of the radio telescope (see Gioia et al. 1982). NGC 4501 and NGC 4654 were also observed at $10.45 \mathrm{GHz}$ with a fourhorn ( $3^{\prime}$ between adjacent horns) system (described by Schmidt et al. 1993). NGC 4654 was measured again at $8.35 \mathrm{GHz}$ with a single horn. Each horn was equipped with two total-power receivers and an IF polarimeter resulting in 4 channels containing the Stokes parameters $I$ ( 2 channels), $Q$, and $U$. The telescope pointing was corrected by performing cross-scans of a bright point source close to the observed galaxy. The flux density scale was established by mapping point sources 3C 138 and 3C 286.

The data reduction was performed using the NOD2 data reduction package (Haslam 1974). At $4.85 \mathrm{GHz}$ and at $10.45 \mathrm{GHz}$ (dual or multibeam systems used), a number of coverages in the azimuth-elevation frame were obtained for each galaxy, as indicated in Table 1. By combining the information from appropriate horns, using the "software beam-switching" technique (Morsi \& Reich 1986) followed by a restoration of total intensities, we obtained for each coverage the $I, Q, U$ maps of the galaxy. The restoration method and its limitations are described in Emerson et al. (1979). At $8.35 \mathrm{GHz}$ (single horn), we performed scans alternatively along the RA and Dec directions, so no restoration was necessary.

All coverages were combined using the spatial frequency weighting method (Emerson \& Gräve 1988) yielding the final maps of total power, polarized intensity, polarization degree, and polarization position angles. To show the structure of the magnetic field projected on the sky plane, we used apparent $\boldsymbol{B}$-vectors defined as $\boldsymbol{E}$-vectors rotated by $90^{\circ}$. In order to remove spatial frequencies corresponding to noisy structures smaller than the beam size, a digital filtering process was applied to the final maps.

In objects showing weak polarized features in the vicinity of a strong unpolarized nuclear source (e.g. NGC 4438, Sect. 3) we used the diagrams of instrumentally polarized signal kindly

\footnotetext{
${ }^{1}$ http://www .mpifr-bonn.mpg.de
} 
supplied by Dr H. Rottmann (see also Rottmann et al. 1996) and converted the instrumental $U$ and $Q$ patterns to the form of maps of polarized intensity, scaled to the same amplitude of total power as the unpolarized source and compared them to our observations, in order to estimate the level of influence of the instrumental polarization on our data (see Sect. 3.1).

\section{Results}

Sections 3.1-3.6 present maps of individual galaxies. Their integrated data are summarised in Table 2 at the end of Sect. 3. The data includes total power and polarized flux densities aquired by integration in polygonal areas encompassing all of the radio emission. From these data, polarization degrees have been obtained, also presented in Table 2.

\subsection{NGC 4438}

NGC 4438 is located close to the cluster centre, about 0.9 (270 kpc in the sky plane) from M87 (Virgo A). It interacts tidally with its close companion NGC 4435 (Combes et al. 1988).

The peak of total power coincides with the position of the galaxy's centre (Fig. 1). The polarization $\boldsymbol{B}$-vectors are oriented here parallel to the disk. The peak of polarized intensity is located, however, outside of the bright star-forming disk (Fig. 2). It is displaced from the optical centre towards the southwest by about $55 \operatorname{arcsec}(4.6 \mathrm{kpc})$. At this position the optical images show a complex of dust lanes that indicate strong compression effects. Other ISM tracers ( $\mathrm{H}$ I, $\mathrm{H} \alpha, \mathrm{CO}, \mathrm{FIR}, \mathrm{X}$-rays) also show emission to the west of NGC 4438 (e.g. Kenney et al. 1995; Machacek et al. 2004; Chemin et al. 2005). We find that most of polarized emission $(\simeq 74 \%)$ comes from the western side of the major axis.

The tail extending towards the southwest visible on both total power and polarized intensity maps (Figs. 1 and 2) is due to a blend of two unresolved background sources, very visible in the map by Condon (1987) at $1.49 \mathrm{GHz}$. These are the NVSS radio sources J122730.6 + 125629 and J122728.5 + 125535 with total flux densities (at $1.4 \mathrm{GHz}$ ) of $38.52 \mathrm{mJy}$ and $19.76 \mathrm{mJy}$, respectively (Condon 1998). Another weak radio source causes the total power extension to the south. It is J122747.6+125647 with total flux density of $2.48 \mathrm{mJy}$ at $1.4 \mathrm{GHz}$.

Taking possible confusion effects into account, we state that NGC 4438 shows evidence of low surface-brightness extraplanar features on either side of the galaxy disk: $\mathrm{RA}_{2000}=12^{\mathrm{h}} 27^{\mathrm{m}} 48^{\mathrm{s}} .5$, $\mathrm{Dec}_{2000}=13^{\circ} 00^{\prime} 59^{\prime \prime}$ and $\mathrm{RA}_{2000}=12^{\mathrm{h}} 27^{\mathrm{m}} 26^{\mathrm{s}}, \mathrm{Dec}_{2000}=$ $13^{\circ} 01^{\prime} 30^{\prime \prime}$. In total power the effect is less pronounced than in polarization because of a considerably higher noise level (Table 1). It is however not due to sidelobe effects. We used the response of the Effelsberg radio telescope to a point source of the same intensity as in NGC 4438 to simulate the map made from a number of coverages with the range of parallactic angles similar to that for this galaxy. Around the main beam response, we obtained a weak sidelobe ring with the brightness of $0.5-0.7 \mathrm{mJy} / \mathrm{b}$.a. This is three times below the level of our first contour in Fig. 1, thus the telescope sidelobes cannot be entirely responsible for the total power extensions in NGC 4438.

The extraplanar extensions are much more visible in polarization (Fig. 2) and extend to at least $25 \mathrm{kpc}\left(5^{\prime}\right)$ distance from the galaxy's optical centre. No emission is visible in these regions in the Condon and NVSS maps. The polarization $\boldsymbol{B}$-vectors are highly inclined to the disk. To check for a possible

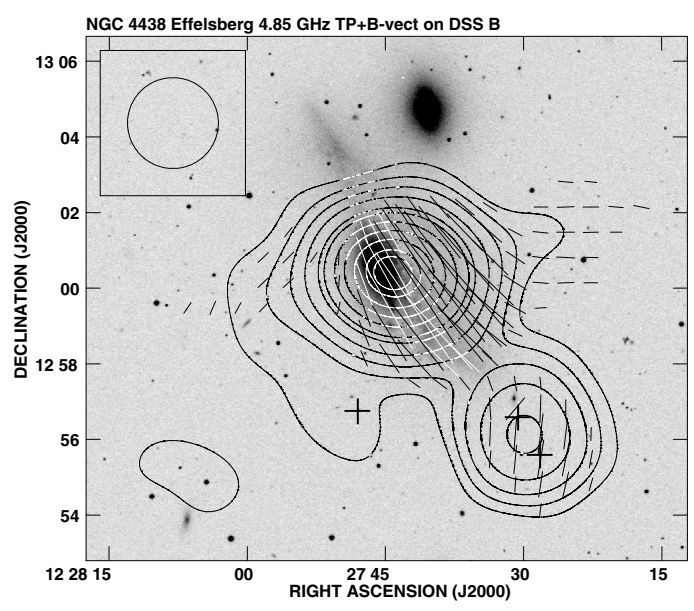

Fig. 1. The total power map of NGC 4438 at $4.85 \mathrm{GHz}$ with apparent $\boldsymbol{B}$-vectors of polarized intensity overlaid onto the DSS blue image. The contours are 3, 6, 10, 16, 25, 30, 40, 50, 60, 70, $75 \times 0.71 \mathrm{mJy} / \mathrm{b}$.a., and a vector of $1^{\prime}$ length corresponds to the polarized intensity of $0.7 \mathrm{mJy} / \mathrm{b} . \mathrm{a}$. The map resolution is 2.5. The companion galaxy NGC 4435 is visible in the north. The beam size is shown in the top left corner of the figure. Crosses indicate the positions of the background sources (see text).

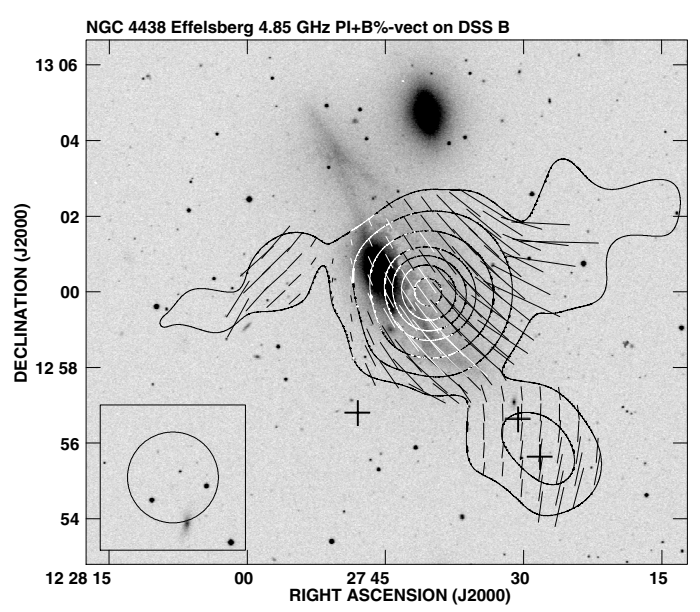

Fig. 2. The map of polarized intensity of NGC 4438 at $4.85 \mathrm{GHz}$ with apparent $\boldsymbol{B}$-vectors of polarization degree overlaid onto the DSS blue image. The contours are 3, 8, 16, 25, 30, 35, $40 \times 0.07 \mathrm{mJy} / \mathrm{b} . \mathrm{a}$., and a vector of $1^{\prime}$ length corresponds to the polarization degree of $7.5 \%$. The map resolution is 2.5 . The beam size is shown in the bottom left corner of the figure. Crosses indicate the positions of the background sources (see text).

contribution of spurious instrumental polarization from a strong, unpolarized central source to these structures, we used the aforementioned instrumental polarization patterns. We compared the cross-sections of observed and instrumentally polarized signal at various position angles. We found that the peaks of instrumental polarization with an amplitude of about $0.3 \mathrm{mJy} / \mathrm{b}$.a. exist at all position angles at the distance of some $70^{\prime \prime}$ (roughly half of the beam), dropping to almost zero just beyond $3^{\prime}$ from the centre. No instrumentally polarized signal stronger than $0.07 \mathrm{mJy} / \mathrm{b} . \mathrm{a}$. ( $1 \sigma$ of the polarized intensity map) has been found at distances greater than one beam size from the centre. In contrast, in NGC 4438 the polarized signal stronger than $3 \sigma$ continues up to 2 beam sizes $\left(5^{\prime}\right)$ away from the centre for position angles between $90^{\circ}$ and $120^{\circ}$. This agrees well with the orientation of extraplanar structures found by Hummel \& Saikia (1991). The apparent $\boldsymbol{B}$-vectors are aligned with the discussed extensions 


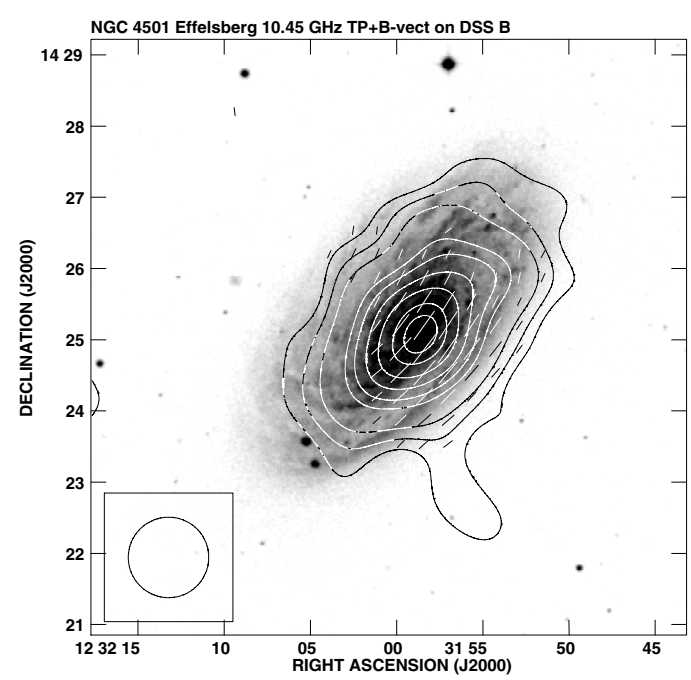

Fig. 3. The total power map of NGC 4501 at $10.45 \mathrm{GHz}$ with apparent $\boldsymbol{B}$-vectors of polarized intensity overlaid onto the DSS blue image. The contours are $3,5,8,16,20,25,30,35,38 \times 0.44 \mathrm{mJy} / \mathrm{b}$.a., and a vector of $1^{\prime}$ length corresponds to the polarized intensity of $5 \mathrm{mJy} / \mathrm{b}$.a. The map resolution is $11^{\prime} 13$. The beam size is shown in the bottom left corner of the figure.

as also found in NGC 4569, another Virgo Cluster galaxy with large-scale outflows (Chyży et al. 2006). For all these reasons we believe that the vertical extensions in NGC 4438 cannot be due to instrumental polarization and are the real structures.

No radio emission stronger than $2 \mathrm{mJy} / \mathrm{b}$.a. in total power and $0.2 \mathrm{mJy} / \mathrm{b}$.a. in polarized intensity has been detected at the position of an S0 companion of NGC 4438 - NGC 4435. This is not surprising: although this galaxy contains some $\mathrm{CO}$ (Vollmer et al. 2005), the mass of molecular gas is 20 times lower than that of NGC 4438. NGC 4435 also shows a very weak $\mathrm{H} \alpha$ emission extending only a few arcsec from the centre (Kenney et al. 1995). This indicates the low content of young stars (see also Boselli et al. 2005) and of X-ray emitting gas (Burstein et al. 1997) produced by supernovae responsible for accelerating the radioemitting cosmic-ray electrons.

\subsection{NGC 4501}

NGC 4501 is located at a distance of $2^{\circ}(0.6 \mathrm{Mpc}$ in the sky plane) from the cluster core. As it is poorly resolved at $4.85 \mathrm{GHz}$, we discuss its radio structure using our observations at $10.45 \mathrm{GHz}$ with almost twice better resolution.

The total power emission at $10.45 \mathrm{GHz}$ from this galaxy (Fig. 3) is symmetric and coincides with the optical disk. The only extension outside the disk at $\mathrm{RA}_{2000}=12^{\mathrm{h}} 31^{\mathrm{m}} 56^{\mathrm{s}}$, $\mathrm{Dec}_{2000}=14^{\circ} 22^{\prime} 30^{\prime \prime}$ is due to a weak background source visible in the NVSS map.

The polarized emission is strongly shifted towards the southwest (Fig. 4), and over $85 \%$ of the polarized flux originates on the SW side of the major axis. The degree of polarization is quite high in this region, reaching some $20 \%$.

\subsection{NGC 4535}

NGC 4535 is a grand-design spiral galaxy located in the southern extension of the Virgo Cluster at the distance of $4.3(1.29 \mathrm{Mpc}$ in the sky plane) from Virgo A.

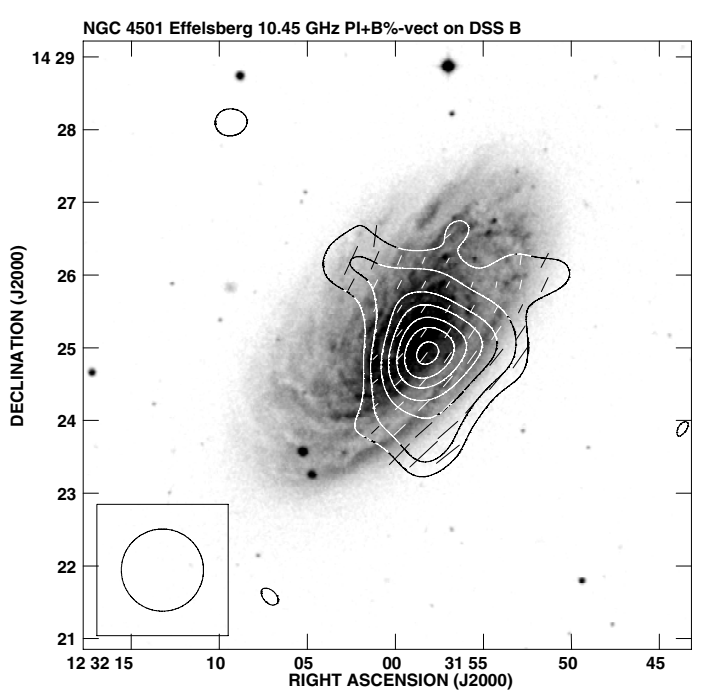

Fig. 4. The map of polarized intensity of NGC 4501 at $10.45 \mathrm{GHz}$ with apparent $\boldsymbol{B}$-vectors of polarization degree overlaid onto the DSS blue image. The contours are 3, 5, 8, 10, 12, 13.5, $15 \times 0.17 \mathrm{mJy} / \mathrm{b} . \mathrm{a}$., and a vector of $30^{\prime \prime}$ length corresponds to the polarization degree of $50 \%$. The map resolution is $11^{\prime} 13$. The beam size is shown in the bottom left corner of the figure.

Optical images show that NGC 4535 has a very regular spiral structure and shows a quite symmetric $\mathrm{H}$ I distribution (Cayatte et al. 1990). The emission in total intensity is distributed in a fairly symmetric manner as well. Its peak is roughly situated at the galaxy's centre (Fig. 5).

The map of polarized intensity reveals a very strong asymmetry with $75 \%$ of the polarized flux coming from the western half of the disk (Fig. 6). The peak of the polarized emission is located outside the optical structure. Polarization $\boldsymbol{B}$-vectors in this structure are generally parallel to the western optical arm of the galaxy. As the total power is distributed symmetrically, the asymmetry in the polarized intensity is due to a gradient in the polarization degree (see Sect. 4.2). The extension to the northeast is due to a background source.

\subsection{NGC 4548}

NGC 4548 is located in the northern part of the Virgo Cluster at a distance of $2.4^{\circ}(0.72 \mathrm{Mpc}$ in the sky plane $)$ from Virgo A. It is an anaemic galaxy showing few signs of star formation, and it is very poor in neutral gas (Cayatte et al. 1990). It shows a generally weak total power emission peaking south of the optical nucleus (Fig. 7). The polarized intensity is only slightly above the noise level (Fig. 8), but the degree of polarization is quite high, reaching $\approx 15 \%$. The polarized emission is concentrated in the central parts of the galaxy and extends along the bar. It coincides with an east-west elongated hole in the H I emission (Vollmer et al. 1999). The polarized emission peaks are on the eastern end of the bar. The observed $\boldsymbol{B}$-vectors are roughly perpendicular to the bar.

\subsection{NGC 4654}

NGC 4654 is located in the eastern part of the cluster, in the area of the eastern subcluster (Gavazzi 1999). Its angular distance from Virgo A is 3.3 (0.99 Mpc in the sky plane). The neutral gas distribution has a comet-like shape (Phookun \& Mundy 1995) with a gaseous tail extending towards the southeast. As it is 
Table 2. Integrated data of studied galaxies.

\begin{tabular}{|c|c|c|c|c|c|c|c|}
\hline NGC & $\begin{array}{l}S_{4.85 \mathrm{GHz}} \\
(\mathrm{TP})[\mathrm{mJy}]\end{array}$ & $\begin{array}{l}S_{4.85 \mathrm{GHz}} \\
(\mathrm{POL})[\mathrm{mJy}]\end{array}$ & $\begin{array}{l}S_{8.35 \mathrm{GHz}} \\
(\mathrm{TP})[\mathrm{mJy}]\end{array}$ & $\begin{array}{l}S_{8.35 \mathrm{GHz}} \\
(\mathrm{POL})[\mathrm{mJy}]\end{array}$ & $\begin{array}{l}S_{10.45 \mathrm{GHz}} \\
\text { (TP) [mJy] }\end{array}$ & $\begin{array}{l}S_{10.45 \mathrm{GHz}} \\
(\mathrm{POL})[\mathrm{mJy}]\end{array}$ & $\begin{array}{l}\% \mathrm{p} \\
-\end{array}$ \\
\hline 4438 & $86.8 \pm 6.5$ & $5 \pm 0.6$ & - & - & - & - & $5.8 \pm 0.8$ \\
\hline \multirow[t]{2}{*}{4501} & $102.4 \pm 8.1$ & $10.5 \pm 1$ & - & - & - & - & $10.3 \pm 1.3^{1}$ \\
\hline & - & - & - & - & $53.5 \pm 5.1$ & $6.8 \pm 1.6$ & $12.8 \pm 3.3^{3}$ \\
\hline 4535 & $39.5 \pm 4.3$ & $4.6 \pm 0.8$ & - & - & - & - & $11.7 \pm 2.3$ \\
\hline 4548 & $5.7 \pm 1.8$ & $0.6 \pm 0.3$ & - & - & - & - & $11.2 \pm 6.2$ \\
\hline \multirow[t]{3}{*}{4654} & $50.2 \pm 4.4$ & $2.2 \pm 0.5$ & - & - & - & - & $4.4 \pm 1^{1}$ \\
\hline & - & - & $34.9 \pm 3$ & $1.4 \pm 0.7$ & - & - & $4 \pm 2.5^{2}$ \\
\hline & - & - & - & - & $24.3 \pm 3.5$ & $1.6 \pm 0.9$ & $6.4 \pm 3.8^{3}$ \\
\hline
\end{tabular}

$\% \mathrm{p}$ - polarization degree.

$\mathrm{TP}=$ total power flux density, $\mathrm{POL}=$ polarized flux density.

${ }^{1}$ At $4.85 \mathrm{GHz} .{ }^{2}$ At $8.35 \mathrm{GHz} .{ }^{3}$ At $10.45 \mathrm{GHz}$.

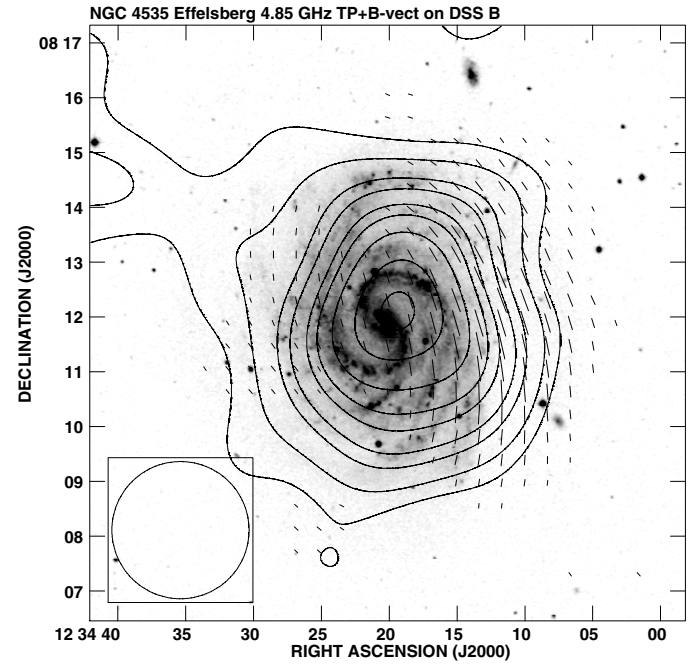

Fig. 5. The total power map of NGC 4535 at $4.85 \mathrm{GHz}$ with apparent $\boldsymbol{B}$-vectors of polarized intensity overlaid onto the DSS blue image. The contours are $3,5,8,10,13,15,18,22,25 \times 0.68 \mathrm{mJy} / \mathrm{b}$.a., and a vector of $1^{\prime}$ length corresponds to the polarized intensity of $3.75 \mathrm{mJy} / \mathrm{b}$.a. The map resolution is 2.5 . The beam size is shown in the bottom left corner of the figure.

poorly resolved at $4.85 \mathrm{GHz}$ and weak at $10.45 \mathrm{GHz}$, we discuss the radio and polarized structures using our observations at $8.35 \mathrm{GHz}$.

The distribution of the total power emission from NGC 4654 resembles the overall shape of the optical image and seems to reflect the star-forming activity in general (Fig. 9). The peak of total power is slightly shifted towards the optically bright clump in the northwestern side of the galaxy visible in the optical images, as well as in the H I maps (see Fig. 10). The polarized emission again has a highly asymmetric distribution. The peak of the polarized brightness is considerably shifted from the disk towards the southwest (Fig. 10). It is located at the base of the aforementioned H I plume extending in the southeastern direction. The $\boldsymbol{B}$-vectors are parallel to the plume instead of following the optical arm that turns up northwards in the southeastern disk.

Recently Soida et al. (2006) presented a high-resolution VLA map of polarized intensity for NGC 4654 at $4.85 \mathrm{GHz}$. It confirms the existence of the polarized feature associated with the H I tail. It also shows a second (weaker) polarization peak in the northwestern disk region. In our Effelsberg map with a lower resolution, the latter is significantly weakened due to beam

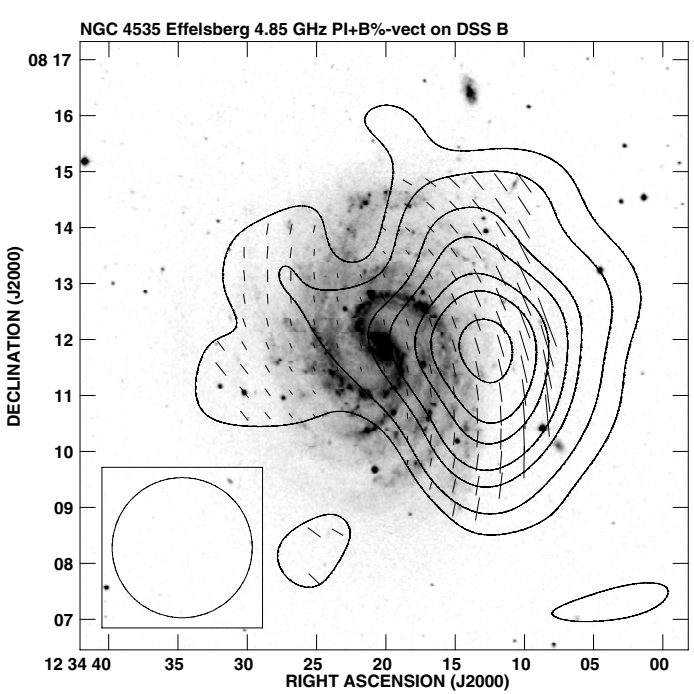

Fig. 6. The map of polarized intensity of NGC 4535 at $4.85 \mathrm{GHz}$ with apparent $\boldsymbol{B}$-vectors of polarization degree overlaid onto the DSS blue image. The contours are $3,5,8,10,13,15,18 \times 0.1 \mathrm{mJy} / \mathrm{b} . \mathrm{a}$., and a vector of $1^{\prime}$ length corresponds to the polarization degree of $60 \%$. The map resolution is 2.5. The beam size is shown in the bottom left corner of the figure.

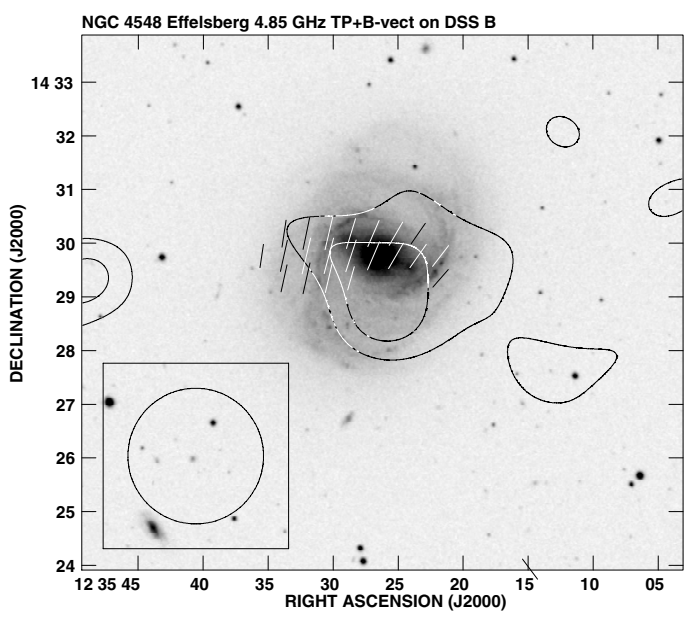

Fig. 7. The total power map of NGC 4548 at $4.85 \mathrm{GHz}$ with apparent $\boldsymbol{B}$-vectors of polarized intensity overlaid onto the DSS blue image. The contours are $3,4 \times 0.77 \mathrm{mJy} / \mathrm{b}$.a., and a vector of $1^{\prime}$ length corresponds to the polarized intensity of $0.84 \mathrm{mJy} / \mathrm{b}$.a. The map resolution is 2.5 . The beam size is shown in the bottom left corner of the figure. 


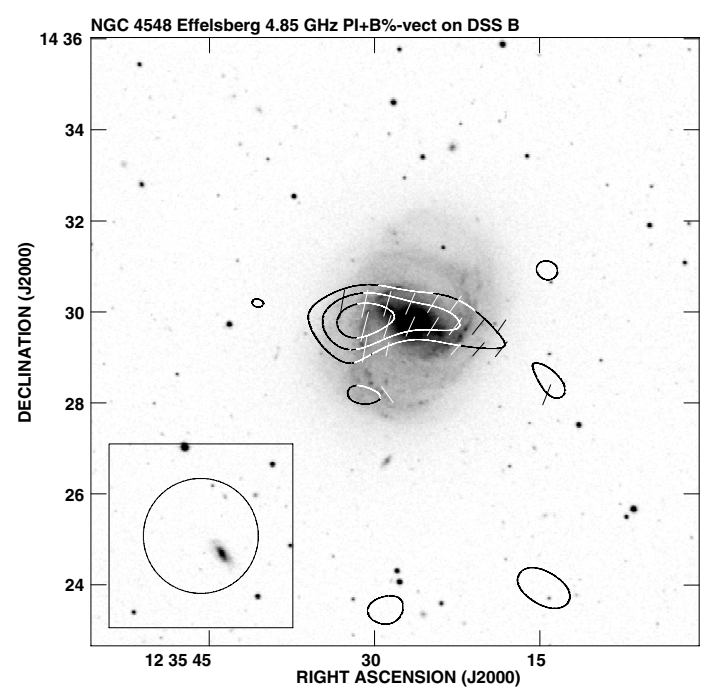

Fig. 8. The map of polarized intensity of NGC 4548 at $4.85 \mathrm{GHz}$ with apparent $\boldsymbol{B}$-vectors of polarization degree overlaid onto the DSS blue image. The contours are 3.5, 4.5, $5.5 \times 0.09 \mathrm{mJy} / \mathrm{b}$.a., and a vector of $1^{\prime}$ length corresponds to the polarization degree of $30 \%$. The map resolution is 2.5 . The beam size is shown in the bottom left corner of the figure.

depolarization effects, as the magnetic field rapidly changes its orientation in this disk part.

\subsection{Faraday rotation}

For both NGC 4501 and NGC 4654, polarization observations at two frequencies were performed, which enables determination of mean Faraday rotation between $4.85 \mathrm{GHz}$ and $10.45 \mathrm{GHz}$. The high-frequency maps were convolved to the resolution at $4.85 \mathrm{GHz}$. The intrinsic Faraday rotation in spiral galaxies varies between positive and negative values that tend to cancel when observed with a large beam. This allows detection possible foreground rotation in the cluster medium.

In the case of NGC 4501, the Faraday rotation measure (RM) changes gradually from +14 to $+46 \mathrm{rad} / \mathrm{m}^{2}$ across the disk in the southeast-northwest direction, with a mean value of $+30 \mathrm{rad} / \mathrm{m}^{2}$. In NGC 4654 we see two distinct areas of moderately high rotation of $57 \mathrm{rad} / \mathrm{m}^{2}$ in the southeastern part of the disk changing to $-57 \mathrm{rad} / \mathrm{m}^{2}--66 \mathrm{rad} / \mathrm{m}^{2}$ on the opposite disk side. The mean RM amounts to only $-4 \mathrm{rad} / \mathrm{m}^{2}$. These extreme values correspond to a maximum rotation of polarization plane by $13^{\circ}$ at $4.85 \mathrm{GHz}$ (either counterclockwise or clockwise). This amount is in fact smaller for most of our data. Several times smaller rotation is expected at higher frequencies. For observations with our large beam, we can use the apparent $\boldsymbol{B}$-vectors as a good approximation of the sky-projected orientation of large scale regular magnetic fields, accurate to some $\pm 10^{\circ}$ at the lowest of our frequencies.

\section{Discussion}

The results shown in Sect. 3 present a variety of effects occurring in cluster galaxies. Below, we make an attempt to categorise various effects according to different physical processes governing the interactions.

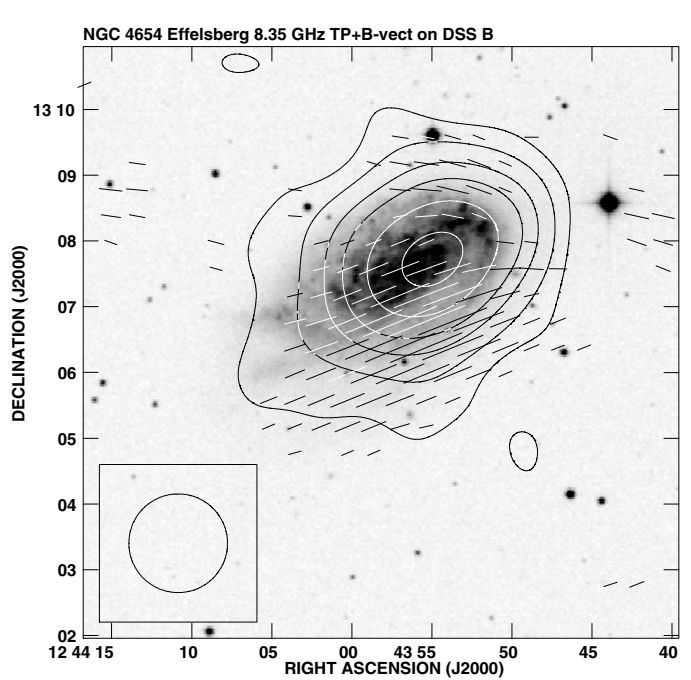

Fig. 9. The total power map of NGC 4654 at $8.35 \mathrm{GHz}$ with apparent $\boldsymbol{B}$-vectors of polarized intensity overlaid onto the DSS blue image. The contours are $3,8,16,25,40,60 \times 0.25 \mathrm{mJy} / \mathrm{b}$.a., and a vector of $1^{\prime}$ length corresponds to the polarized intensity of $0.4 \mathrm{mJy} / \mathrm{b}$.a. The map resolution is 1'.5. The beam size is shown in the bottom left corner of the figure.

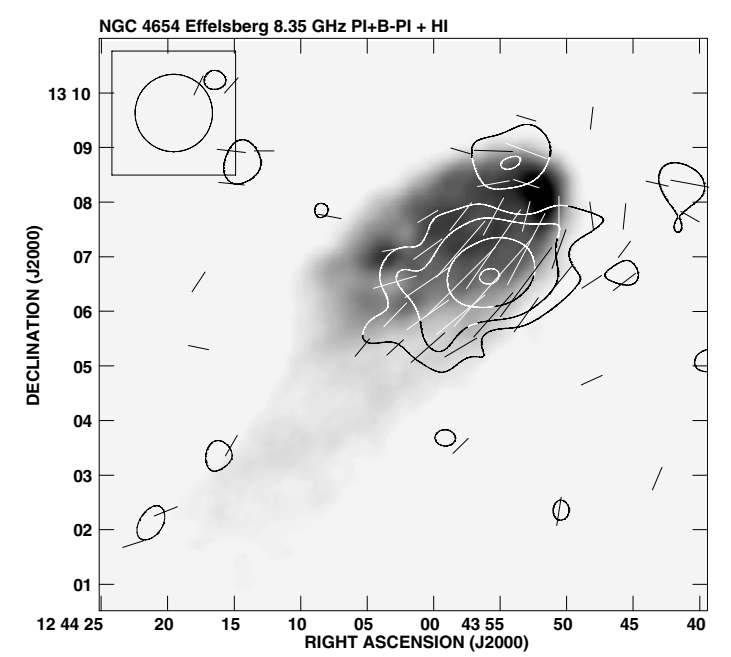

Fig. 10. The map of polarized intensity of NGC 4654 at $8.35 \mathrm{GHz}$ with apparent $\boldsymbol{B}$-vectors of polarization degree overlaid onto the $\mathrm{HI}$ image (Phookun \& Mundy 1995). The contours are 3.5, 5, 8, 13, 18, $21 \times$ $0.03 \mathrm{mJy} / \mathrm{b} . \mathrm{a}$., and a vector of $1^{\prime}$ length corresponds to the polarization degree of $12.5 \%$. The map resolution is 1.5 . The beam size is shown in the top left corner of the figure.

\subsection{Galactic anaemia}

NGC 4548 (Figs. 7 and 8), the only anaemic galaxy in our sample has no close companions and shows an H I deficiency of 0.76 (Koopmann \& Kenney 2004; Cayatte et al. 1994). It is a very weak radio source. The integrated flux density (hence total luminosity) at $4.85 \mathrm{GHz}$ is by a factor of 6-10 times lower than that of other spirals in our sample. It is also several times lower than the total flux density of $54 \mathrm{mJy}$ (roughly half of it coming from the disk region - Gallimore et al. 2006) measured at this frequency by Becker et al. 1991 for NGC 4579 - another Virgo Cluster object of similar morphological type and H I deficiency (Cayatte et al. 1994). This makes NGC 4548 an exceptionally weak radio emitting galaxy, even for an anaemic spiral. 


\subsection{Ram pressure effects - compression}

Otmianowska-Mazur \& Vollmer (2003) show that the magnetic perturbations in the outer disk may last $0.7-0.9$ Gyr after the passage through the dense ICM. Within this time galaxies travelling at the speed of over $1000 \mathrm{~km} \mathrm{~s}^{-1}$ with respect to the ICM (many Virgo Cluster spirals move faster) might have moved by almost $1 \mathrm{Mpc}$ (considerably more than $3^{\circ}$ in case of transverse motions) from the cluster core. Thus, the observed signatures of magnetic field compression may either have occurred recently or they may constitute a sensitive, long-lasting memory of the epoch (some hundreds Myr ago) when the galaxy was much closer to the Virgo Cluster centre. These two scenarios are presently difficult to distinguish.

There are clear examples of galaxies in our sample that show primary importance of ram pressure effects, without any influence of tidal interactions. The first of them is NGC 4501 (Figs. 3 and 4), in which the asymmetry of polarized emission is significant with more than $85 \%$ of the polarized emission found southwest of the major axis. This is unusual for normal spirals and may stem from the enhancement of regular and/or random, but anisotropic, magnetic fields by compression as described in detail by Beck et al. (2005). If the enhancement results from compression in the sky plane, both these magnetic field structures may yield high polarization. We note that distinguishing between unidirectional and anisotropic random magnetic fields needs Faraday rotation data with an appropriate resolution.

Our beam is too large to decide whether we are dealing with a narrow compressional ridge here. We note, however, that the maximum of the polarized flux density occurs on the disk side where strong gradients of $\mathrm{HI}$ distribution were observed (Cayatte et al. 1990), and a bright spot of X-ray emission can be seen (Böhringer et al. 1994). Moreover, the optical structure also shows a smaller pitch angle on this disk side than on the opposite side. This asymmetry resembles that in NGC 4254, in which compression effects were suggested (Chyży et al. 2002). The latter object, in which the VLA data reveal a narrow compressional ridge, shows only a global asymmetry of the polarized brightness when observed at a resolution similar to that for NGC 4501 (Soida et al. 1996). The explanation of the asymmetry of NGC 4501 by ram pressure stripping is supported by its underabundance of $\mathrm{HI}$. The deficiency parameter of 0.34 (Koopmann \& Kenney 2004) places this galaxy among objects suffering significant stripping effects.

The H I data by Cayatte et al. (1990) indicate that the northwestern half of the disk of NGC 4501 is approaching us. If the spiral is trailing this would mean that the compressed edge is the remote one and the galaxy is viewed "from below" (see also Onodera et al. 2004). The compression along the remote edge agrees well with a high positive velocity of NGC 4501 with respect to M87 and to the average velocity for other galaxies in this Virgo Cluster region (LEDA database). The excess of polarization occurs in the "head-on" disk side and can be attributed to a compressional enhancement of regular or random and anisotropic magnetic fields.

Another example of ram pressure effects is NGC 4535 (Figs. 5 and 6), which has symmetric, grand-design distribution of stars. In this case the peak of polarized intensity is located outside the bright optical spiral arms, while the total power emission is as symmetric as the optical structure. The asymmetry in the distribution of polarized intensity is clearly caused by an eastwest gradient in the degree of magnetic field ordering. In fact, the mean polarization degree (integrated in areas of roughly $5^{\prime} \times 5^{\prime}$ located east and west of the north-south line going through the centre) is only $6 \%$ in the eastern disk half rising to $16 \%$ in the western one. This asymmetry is surprising because NGC 4535 is located in the southern Virgo extension (Böhringer et al. 1994), where the intracluster gas density is rather moderate. As noted in the beginning of this section, we cannot exclude the possibility that the distortion may have occurred some hundreds of Myrs ago (see Otmianowska-Mazur \& Vollmer 2003). In this case strong magnetic anomalies accompanying only weak perturbations observed in the HI gas (Cayatte et al. 1990) may imply that the magnetic fields provide a very long-lasting memory of past interactions, possibly caused by a back-falling gas stripped some hundreds of Myrs ago. On the other hand, if NGC 4535 is being stripped now, it may be moving in the sky plane from east towards the west. This information is impossible to acquire from radial velocity studies alone. The latter possibility would also mean that the magnetic field structure may respond strongly to even quite weak ram pressure efects.

\subsection{Tidal interactions mixed with ram pressure effects}

Gravitational encounters may play a significant role in the evolution of cluster spirals as the spatial density of objects is high. However, in the cluster centre, disruptive encounters are rare as high relative velocities of galaxies make the encounters very short; as shown by numerical simulations (Soida et al. 2006; Vollmer et al. 2005), typical timescales for developing the observed signatures of tidal interactions (with some admixture of ram pressure) involve about $500 \mathrm{Myr}$ for loose encounters in the Virgo Cluster outskirts and about 150 Myr close to the Cluster core. Strong gravitational effects may result in enhanced nuclear activity (either LINER, Seyfert-type, or nuclear starburst) triggered by highly non-circular motions. General distortions of the structure may occur, and those involving the stellar component may constitute an unambiguous signature of tidal effects. At the same time perturbations of the gaseous disk and of the magnetic field structure may develop, since they are often accompanied by magnetized gaseous tails that might provide additional effects. There are two galaxies in our sample that show evidence of tidal perturbations, as well as revealing ram pressure effects exerted by the cluster medium. We discuss these cases in detail.

\subsubsection{NGC 4438}

The asymmetrically disturbed optical morphology of NGC 4438, as well as observations in CO (Combes et al. 1988; Vollmer et al. 2005), $\mathrm{H} \alpha$ (Kenney et al. 1995, Chemin et al. 2005), and X-rays (Machacek et al. 2004) clearly suggest tidal interactions with the companion galaxy NGC 4435. Furthermore, the X-ray properties of the gas outside the nuclear regions of these galaxies trace their past collision (Machacek et al. 2004).

NGC 4438 is the only galaxy in our small sample showing a strong central source. As it is also of earliest Hubble type (Sa), it has the strongest mass concentration (hence deepest gravitational potential minimum) in the centre. The VLA A and B-array observations of Hummel \& Saikia (1991, resolution 0.'4-2') show that about $30 \%$ of the total flux density at $4.86 \mathrm{GHz}$ comes from an arcsecond-scale compact radio shell surrounding the nucleus, classified as a LINER (NED database). They also detected radio extensions (up to $10^{\prime \prime}$ ) perpendicular to the galaxy plane. Such nuclear activity can possibly be triggered by tidal encounters. Our observations indeed reveal a strong total intensity peak at the position of the galaxy centre (Fig. 1). The recent D-array VLA map at $4.86 \mathrm{GHz}$ by Vollmer et al. (2007) has a lower 


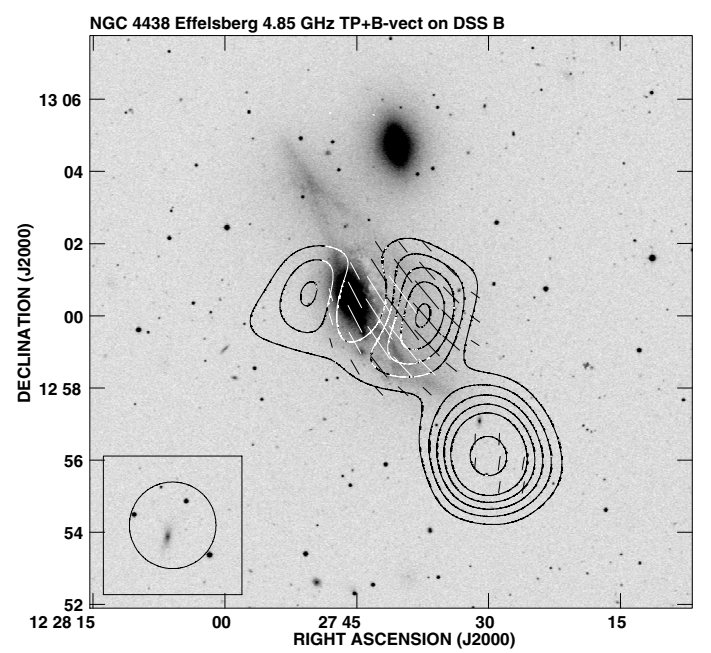

Fig. 11. The residual total power brightness after the "maximum subtraction" of the central source with $\boldsymbol{B}$-vectors for the brightest polarized peak, both overlaid onto the blue DSS image. The contour levels are 6, $9,12,15,18,25 \times 0.7 \mathrm{mJy} /$ b.a., and the polarization vectors are truncated at the level of $0.5 \mathrm{mJy} / \mathrm{b} . \mathrm{a}$. A vector of $1^{\prime}$ length corresponds to $2 \mathrm{mJy} / \mathrm{b} . \mathrm{a}$.

resolution of $18^{\prime \prime}$ but recovers almost all the extended flux seen by us with the Effelsberg radio telescope. This map shows that all structures inside central $18^{\prime \prime}$ of NGC 4438 account for no more than $50 \%$ of the total galaxy flux density detected in our singledish data.

The map size $\left(20^{\prime \prime}\right)$ in Hummel \& Saikia is too small to observe the (probably extended) polarized peak seen in our singledish observations, shifted by almost $1^{\prime}$ westwards from the centre. Our resolution is too low to definitely decide whether the shift has an external origin - either tidal interactions or ram pressure effects. The "maximum subtraction method" (Chyży et al. 2003) reveals a strong total power residual (peak of $\approx 15 \mathrm{mJy} /$ b.a.) southwest of the nucleus, displaced from the centre in the same direction (but slightly farther out) as the polarized blob (Fig. 11). We checked that it is not due to a (possibly) nonGaussian Effelsberg beam and is not a sidelobe effect. A residual total power peak has been found in the same place by Kotanyi \& Ekers (1983), who claimed it is due to either external compression or tidal interactions. Our residual source is thus real, polarized with $\boldsymbol{B}$-vectors parallel to the disk and may be associated with extraplanar dust, $\mathrm{H} \alpha$, and $\mathrm{X}$-ray structures.

The total power residual possibly associated with the polarized peak that has a disk-parallel magnetic field might be explained by cluster ram pressure compressing the magnetic field. On the other hand, the tidal processes could stretch the magnetic field and enhance the polarized emission, as well as align magnetic fields with extraplanar optical filaments parallel to the disk. Sensitive polarimetric observations with a resolution of $\approx 15^{\prime \prime}-20^{\prime \prime}$ are necessary to evaluate contributions from both processes.

With the high sensitivity to extended structures, our polarization map also reveals weak extended emission on both sides of the disk extending up to $5^{\prime}$ and proven not to be due to instrumental polarization (Sect. 3.1). These polarized features have an integrated intensity of $1.1 \pm 0.1 \mathrm{mJy}$ and are associated neither with optical filaments nor known background sources. The magnetic field vectors have a different orientation here than in the disk region or in the strong western extension and are highly inclined to the disk.
If these extensions are the large-scale magnetized outflows, they must have a pressure and energy density high enough to overcome the ram pressure in the surrounding cluster medium. As suggested by Vollmer et al. (2005), the ambient kinetic pressure in the vicinity of NGC 4438 is $\simeq 10^{-12} \mathrm{~g} \mathrm{~cm}^{-1} \mathrm{~s}^{-2}$. Though our resolution is modest, an assumption that the extraplanar radio features fill our beam can provide the minimum possible values of magnetic and cosmic ray pressures. By assuming the condition of pressure balance between the magnetic field and cosmic rays, we obtain the minimum strength of a stable magnetic field in the outflows to be $3-3.5 \mu \mathrm{G}$. Thus the joint pressure of magnetic fields and cosmic rays in these features must be at least about $10^{-12} \mathrm{~g} \mathrm{~cm}^{-1} \mathrm{~s}^{-2}$ (likely to be considerably higher). For these calculations we adopted the low-energy cosmic ray cutoff at $300 \mathrm{MeV}$, the nonthermal spectral index of 1.0 (similar to that in outflows in NGC 4569, Chyży et al. 2006) and a pathlength of some $10 \mathrm{kpc}$ along the line of sight. If the extraplanar structures are better collimated, the magnetic and cosmic ray pressures become even several times higher. Thus the outflows have enough power to overcome the intracluster gas pressure. Sensitive Effelsberg observations at higher frequencies are desirable to determine the detailed structure and the spectral index of these possible outflows and, thus, to discover their physical nature.

\subsubsection{NGC 4654}

Another galaxy in which tidal forces may at least play some role is NGC 4654, which is probably interacting with the neighbouring NGC 4639. However, the cometary shape of its H I distribution and the gaseous tail extending towards the southwest may be caused by ram pressure effects as the galaxy moves through the ICM (Phookun \& Mundy 1995). The galaxy shows no strong central source, probably because of less pronounced central mass concentration as expected for its much later type $(\mathrm{Sc})$ than NGC 4438. We note that the $\boldsymbol{B}$-vectors clearly follow the tail (as expected for its outflowing motion), rather than the spiral pattern in this part of the disk. Recent simulations of the magnetic field of this galaxy (Vollmer et al. 2003, confirmed by Soida et al. 2006) suggest that a mixture of tidal interaction with some ram pressure effects is the best explanation of the radio polarization structure of NGC 4654. In particular, the bright polarized blob in the western part of the gaseous tail with the magnetic field stretched along the tail, as well as the weaker polarization spot northwest of the centre are well-reproduced.

\subsection{Global properties}

To compare the integrated total power emission from the Virgo Cluster spirals to non-cluster nearby spiral galaxies we analyse the radio - far-infrared (FIR) correlation. We used the distanceindependent quantities as statistical variables: the radio and FIR surface brightness. They are computed by dividing the integrated flux density at $4.85 \mathrm{GHz}$ and at $60 \mu \mathrm{m}$, respectively, by their face-on corrected observed disk surface. For that purpose we used the extinction-corrected diameters from the LEDA database. As a homogenous reference sample, we took the galaxies measured at $4.8 \mathrm{GHz}$ by Gioia et al. (1982) who used the same instrument and a similar method of integrating the total power flux density. The reference sample has been expanded towards low intensities using the $4.85 \mathrm{GHz}$ Effelsberg measurements of low surface-brightness galaxies (Chyży et al. 2007). 


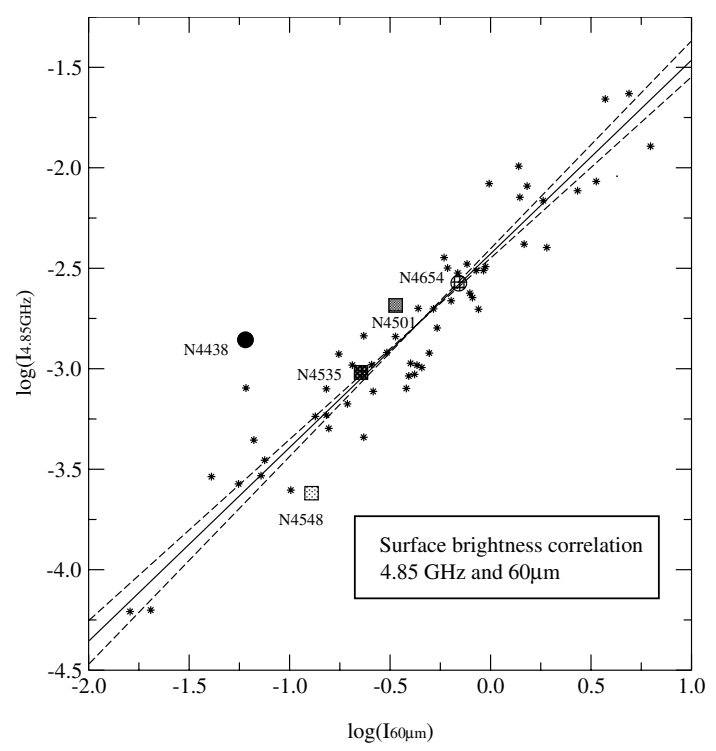

Fig. 12. The radio - FIR correlation diagram for our Virgo objects plotted as symbols with labels and for the reference sample of galaxies observed by Gioia et al. (1982) with an extension towards low surfacebrightness objects observed by Chyży et al. (2007) - both as dots. The surface brightness at $4.85 \mathrm{GHz}\left(\mathrm{Jy} / \square^{\prime}\right)$ and at $60 \mu \mathrm{m}\left(\mathrm{Jy} / \square^{\prime}\right.$, see text $)$ is used. The solid curve is an orthogonal fit to reference galaxies with a slope of $0.96 \pm 0.06$. The dashed lines show the "regression scissors": maximum and minimum slope (1.03 and 0.90$)$ allowed by the data scatter.

The flux densities at $60 \mu \mathrm{m}$ were taken from Helou \& Walker (1995) and, if not present there, from Moshir et al. (1990).

The radio-FIR correlation for our Virgo sample and the reference sample is shown in Fig. 12. Three normally star-forming galaxies: NGC 4501, NGC 4535, and NGC 4654, closely agree with the relation derived for non-Virgo spirals, which has a slope of $0.96 \pm 0.06$. In contrast, NGC 4438 lies considerably above this relation. To check for the significance of this deviation, we computed the rms deviation of the radio brightness from the orthogonal fit shown in Fig. 12. We obtain $0.17 \log \left(\mathrm{Jy} / \square^{\prime}\right)$, thus NGC 4438 deviates significantly by $4.4 \sigma$ from the correlation for normal spirals. The deflection corresponds to an excess of radio over FIR emission by a factor of about 5-5.5. An abnormally high radio/FIR ratio has also been found for this galaxy by Niklas et al. (1995). A similar enhancement of radio emission in Coma Cluster spirals was attributed to compression of magnetic fields caused by the ambient medium by Gavazzi (1998).

The excess could be due either to the western polarized radio peak (Fig. 11), probably associated with some extraplanar radio emission (Kotanyi \& Ekers 1983), or to nonthermal structures unrelated to star formation originating in the nuclear region. According to the VLA map at $4.85 \mathrm{GHz}$ by Vollmer et al. (2007), the central region (innermost $18^{\prime \prime}$ ) of NGC 4438 contributes less than $50 \%$ to the total flux density at $4.85 \mathrm{GHz}$, while the images from the ISO archive show this region to be the source of the bulk of FIR emission. On the other hand, the extraplanar radio source of Kotanyi \& Ekers (1983) emitting much less in FIR amounts to only some $10-12 \%$ of the total flux at $1.4 \mathrm{GHz}$, probably the same fraction at $4.85 \mathrm{GHz}$. Altogether, this is too little to be an excess of a factor of 5 , thus there could be another component undetected by interferometers and not related to star formation, contributing to the excess of radio over FIR emission. To establish a possible association of the excess with circumnuclear or extraplanar structures or to say whether we are dealing with compressional enhancement of magnetic fields as proposed by Reddy \& Yun (2004), we need a substantially better resolution, together with enough sensitivity to extended structures. We note finally that a similar excess of radio over FIR emission for cluster spirals has been noted by Gavazzi (1998).

NGC 4548 has a lower radio surface-brightness than any galaxy in the sample of Gioia et al. (1982). It also belongs to the weakest FIR emitters in that sample. Compared to another radio-bright $\mathrm{Sb}$ type Virgo Cluster spiral, NGC 4501, the discussed galaxy is redder both in $(U-B)_{c}$ and $(B-V)_{c}$ (extinction corrected values from LEDA) by about 1 . 2 . It is located at the extreme red end of the $U-B-B-V$ colour distribution of more than $120 \mathrm{Sb}$ field galaxies brighter than 14.5 selected from the LEDA database. NGC 4548 is obviously deficient in massive stars producing cosmic rays electrons (responsible for the radio emission) via supernova explosions. Its weak radio emission compared to normal spirals is therefore largely due to a low star-formation level caused by the "anaemia", causing its low position on the radio-FIR correlation.

Additionally, NGC 4548 shows some weak negative deviation from the correlation line for normal spirals (Fig. 12). Thus, there is some evidence that the radio emission is even weaker for its FIR brightness than expected. However, the deviation amounts to $-1.9 \sigma$ of the regression residuals. Therefore, this anaemic galaxy shows only weak evidence for an additional deficit of radio emission (hence of total magnetic fields) regarding to what is expected from its low star formation level.

\subsection{Intracluster Faraday rotation}

The measurements of the Faraday rotation of a diffuse emission from the Perseus Cluster (de Bruyn \& Brentjens 2005) or of background sources shining through a number of Abell clusters (Clarke et al. 2001) indicate the existence of microgauss regular magnetic fields in the intracluster medium. They yield Faraday rotations reaching $\pm 40 \mathrm{rad} / \mathrm{m}^{2}$ out to projected distances of $0.5-1 \mathrm{Mpc}$ from the cluster core. Thus, the Faraday rotation effects observed by us may be at least partly due to the intracluster medium. Too far-reaching conclusions from only two objects are rather dangerous; nevertheless, we note that the distance modulus of NGC 4501 determined by Gavazzi et al. (1999) places it at the mean distance of the surrounding part of the Virgo Cluster, while NGC 4654 lies in the front of the eastern subcluster. This would mean that NGC 4501 may shine through at least half of the thickness of the magnetized intracluster gas, while NGC 4654 lies in the foreground of the cluster medium. However, it is too early to conclude that the differences in mean Faraday rotation (Sect. 3.6) reflect the position of the two galaxies with respect to the magnetized intracluster gas. That the above estimate of the distance to NGC 4654 was questioned by Soida et al. (2006) raises even more doubts. Such questions will be the subject of future observational work using a much larger sample that also includes background objects.

\section{Summary and conclusions}

We present results of the first systematic study of the magnetic field structures of Virgo Cluster spirals. We used the Effelsberg radio telescope at $4.85 \mathrm{GHz}$ to detect weak extended total power and polarized emission, while the radio-brightest objects were studied in more detail at $8.35 \mathrm{GHz}$ or at $10.45 \mathrm{GHz}$. The results are as follows:

- Ram pressure effects mainly influence the gaseous component. NGC 4501, located in a relatively dense hot gas 
environment, shows a typical wind-swept Hi asymmetry (and some weak optical effects), while NGC 4535, surrounded by a more dilute hot medium, lacks clear signs of interaction in the optical domain and in the neutral gas distribution. Nevertheless, both objects show a strong concentration of polarized emission on one disk side. In NGC 4501 it agrees with $\mathrm{H}$ I asymmetry and with the direction of movement of the galaxy with respect to the cluster. In the case of NGC 4535, a strong asymmetry of the magnetic field structure occurs at a much lower level of ram pressure effects, providing a clue to the galaxy's motion in the sky plane. Alternatively, NGC 4535 may still "remember" past stripping events.

- Two galaxies with obvious signs of tidal interactions mixed with ram pressure effects behave in a different way: the Satype NGC 4438 shows a polarized peak shifted away from the disk towards the west, situated in a region suspected of external compression and associated with extraplanar emission in $\mathrm{H} \alpha$ and X-rays. The galaxy has a strong, compact central source with clear signs of low surface-brightness magnetized outflows possibly connected to it (detectable only with a single-dish instrument), reaching some $25 \mathrm{kpc}$ away from the galaxy plane. Another object less affected by ram pressure, the Sc-type spiral NGC 4654, has no central source but shows a magnetized H I tail that is best modeled by a combination of tidal forces and ram pressure effects.

- The lack of gas and a low star-formation level in the anaemic galaxy NGC 4548 results in weak radio emission, although this may not be the case for other similar anaemic spirals of a similar Hubble type (e.g. NGC 4579).

- Three out of five galaxies follow the radio $(4.85 \mathrm{GHz})-$ far infrared $(60 \mu \mathrm{m})$ correlation derived for non-cluster spirals closely. In contrast, NGC 4438 shows a strong excess of nonthermal radio emission. The circumnuclear structures are not sufficient to explain this phenomenon, but the compressional enhancement of magnetic fields is a likely cause. On the other hand, the anaemic spiral NGC 4548 shows a marginally significant total power deficiency compared to its FIR emission.

- Faraday rotation measurements are only available for two galaxies: NGC 4501 and NGC 4654. The former is probably located deeper inside the intracluster gas and definitely has a higher mean Faraday rotation than the latter known to lie in the front of its local subcluster. To attribute this effect to the intracluster magnetic fields, a considerably larger sample of cluster galaxies and background objects is needed.

Despite modest resolution, the "magnetic diagnostics" was proven to trace the effects of interactions with other galaxies or with the intracluster gas by providing a very long-lasting memory of past interactions even when effects are weakly visible in other species. Single-dish observations were particularly helpful in the case of radio-weak outflows in NGC 4438. Keeping the promising results of this study in mind, we plan for the near future more systematic studies involving larger samples of cluster spirals and background sources.

Acknowledgements. Three of us: MU, MS, and KCh, are indebted to Professor Richard Wielebinski from the Max-Planck-Institut für Radioastronomie (MPIfR) in Bonn for his multiple invitations to MPIfR, where a large part of this work was done. We thank Dr Elly Berkhuijsen from the Max-Planck-Institut für Radioastronomie (MPIfR) in Bonn for her valuable comments and critical remarks. We would also like to thank the referee, Michael Dumke, for his substantial remarks. This work was supported by the Polish Ministry of Science and
Higher Education, grant 2693/H03/2006/31, and by the Polish-French (ASTROLEA-PF) cooperation program. We acknowledge the use of the HyperLeda database (http://leda.univ-lyon1.fr). The research also used the NRAO VLA Sky Survey (NVSS).

\section{References}

Beck, R., Fletcher, A., Shukurov, A., Snodin, A., et al. 2005, A\&A, 444, 739 Becker, R. H., White, R. L., \& Edwards, A. L. 1991, ApJS, 75, 1 Boselli, A., Gavazzi, G., Lequeux, J., et al. 1997, A\&A, 327, 522 Boselli, A., Boissier, S., Cortese, L., et al. 2005, ApJ, 623, L13 Böhringer, H., Briel, U. G., Schwarz, R. A., et al. 1994, Nature, 368, 828 de Bruyn, A. G., \& Brentjens, M. A. 2005, A\&A, 441, 931 Burstein, D., Jones, C., Forman, W., et al. 1997, ApJS, 111, 163 Byrd, G., \& Valtonen, M. 1990, ApJ, 350, 89

Cayatte, V., van Gorkom, J. H., Balkowski, C., \& Kotanyi, C. 1990, AJ, 100, 604 Cayatte, V., Kotanyi, C., Balkowski, C., \& van Gorkom, J. H. 1994, AJ, 107, 1003

Chemin, L., Cayatte, V., Balkowski, C., et al. 2005, A\&A, 436, 469 Chemin, L., Balkowski, C., Cayatte, V., et al. 2006, MNRAS, 366, 812 Chyży, K. T., Urbanik, M., Soida, M., \& Beck, R. 2002, Ap\&SS, 281, 409 Chyży, K. T., Knapik, J., Bomans, D. J., et al. 2003, A\&A, 405, 513 Chyży, K. T., Soida, M., Bomans, D. J., et al. 2006, A\&A, 447, 465 Chyży, K. T., Bomans, D. J., Krause, M., et al. 2007, A\&A, 462, 933 Clarke, T. E., Kronberg, P. P., \& Böhringer, H. 2001, ApJ, 547, L111 Combes, F., Dupraz, C., Casoli, F., \& Pagani, L. 1988, A\&A, 203, L9 Condon, J. J. 1987, ApJS, 65, 485

Condon, J. J., Cotton, W. D., Greisen, E., et al. 1998, AJ, 115, 1693 Emerson, D. T., \& Gräve, R. 1988, A\&A, 190, 353

Emerson, D. T., Klein, U., \& Haslam, C. G. T. 1979, A\&A, 76, 92 Gallimore, J. F., Axon, D. J., O’Dea, C. P., et al. 2006, AJ, 132, 546

Gavazzi, G. 1998, in Untangling Coma Berenices: A New Vision of an Old Cluster, ed. A. Mazure, F. Casoli, F. Durret, \& D. Gerbal (Word Scientific Publishing Co Pte Ltd), 73

Gavazzi, G., Boselli, A., \& Kennicutt, R. 1991, AJ, 101, 1207

Gavazzi, G., Catinella, B., Carrasco, L., et al. 1998, AJ, 115, 1745

Gavazzi, G., Boselli, A., Scodeggio, M., et al. 1999, MNRAS, 304, 595

Gioia, I. M., Gregorini, L., \& Klein, U. 1982, A\&A, 116, 164

Gunn, J. E., \& Gott, J. R. 1972, ApJ, 176, 1

Haslam, C. G. T. 1974, A\&AS, 15, 333

Helou, G., \& Walker, D. W. 1995, IRAS Small Scale Structure Catalog, Ver 1.00 Vizier Online Data Catalog: VII/73

Hummel, E., \& Saikia, D. J. 1991, A\&A, 249, 43

Kenney, J. D. P., Rubin, V. C., Planesas, P., \& Young, J. S. 1995, ApJ, 438, 135

Kotanyi, C. G., \& Ekers, R. D. 1983, A\&A, 122, 267

Koopmann, R. A., \& Kenney, J. D. P. 2004, ApJ, 613, 866

Machacek, M. E., Jones, C., \& Forman, W. R. 2004, ApJ, 610, 183

Moore, B., Lake, G., \& Katz, N. 1998, ApJ, 495, 139

Morsi, H. W., \& Reich, W. 1986, A\&A, 163, 313

Moshir, M., Copan, G., Conrow, T., et al. 1990, IRAS Faint Source Catalogue, version 2.0, Vizier Online Data Catalog, 2156, 0

Niklas, S., Klein, U., \& Wielebinski, R. 1995, A\&A, 293, 56

Onodera, S., Koda, J., Sofue, Y., \& Kohno, K. 2004, PASJ, 56, 439

Otmianowska-Mazur, K., \& Vollmer, B. 2003, A\&A, 402, 879

Paturel, G. 2003, A\&A, 412, 45

Phookun, B., \& Mundy, L. G. 1995, ApJ, 453, 154

Reddy, N. A., \& Yun, M. S. 2004, ApJ, 600, 695

Rottmann, H., Mack, K.-H., \& Klein, U. 1996, Astron. Ges. Abstract Ser., 12, 223

Schmidt, A., Wongsowijoto, A., Lochner, O., et al. 1993, MPIfR Technical Report No. 73, MPIfR, Bonn

Soida, M., Otmianowska-Mazur, K., Chyży, K. T., \& Vollmer, B. 2006, A\&A, 458,727

Soida, M., Urbanik, M., \& Beck, R. 1996, A\&A, 312, 409

Soida, M., Urbanik, M., Beck, R., et al. 2001, A\&A, 378, 40

Toomre, A., \& Toomre, J. 1972, ApJ, 178, 623

Urbanik, M. 2005, in The Magnetized Plasma In Galaxy Evolution, ed. K. T. Chyży, K. Otmianowska-Mazur, M. Soida, \& R.-J. Dettmar

Valluri, M. 1993, ApJ, 408, 57

Vollmer, B. 2003, A\&A, 398, 525

Vollmer, B., Cayatte, V., Boselli, A., et al. 1999, A\&A, 349, 411

Vollmer, B., Cayatte, V., Balkowski, C., \& Duschl, W. J. 2001, ApJ, 561, 708

Vollmer, B., Balkowski, C., Cayatte, V., et al. 2004a, A\&A, 419, 35

Vollmer, B., Beck, R., Kenney, J. D. P., \& van Gorkom, J. H. 2004b, AJ, 127, 3375

Vollmer, B., Braine, J., Combes, F., \& Sofue, Y. 2005, A\&A, 441, 473

Vollmer, B., Soida, M., Beck, R., et al. 2007, A\&A, 464, 37 